\title{
Sistema de Información del Banco de Tierras de Galicia
}

\author{
Juan Porta ${ }^{1}$, Jorge Parapar ${ }^{1}$, Paula García ${ }^{1}$, Gracia Fernández ${ }^{1}$, Juan Touriño ${ }^{1}$, \\ Francisco Ónega ${ }^{2}$, Pablo Díaz ${ }^{2}$, David Miranda ${ }^{2}$, Rafael Crecente ${ }^{2}$ \\ juan.porta@udc.es,jparaparl@udc.es, pgarciab@udc.es, gfernandez@udc.es, juan@udc.es, \\ franciscojose.onega@usc.es, pablo.diaz.redondo@usc.es, david.miranda@usc.es, \\ rafael.crecente@usc.es \\ ${ }^{1}$ Grupo de Arquitectura de Computadores, Universidad de A Coruña. España. \\ ${ }_{2}^{2}$ Laboratorio del Territorio, Universidad de Santiago de Compostela. España.
}

DOI: $10.4304 /$ risti.9.27-41

Resumen: Este artículo describe el desarrollo del sistema de información del Banco de Tierras de Galicia, organismo gallego que actúa de intermediario entre propietarios y agricultores para fomentar el arrendamiento de tierras y evitar su abandono. Sus objetivos son realizar la gestión interna del Banco, difundirlo entre el público y facilitar distintos tipos de tramitaciones a través de Internet. El sistema, disponible en http://www.bantegal.com/sitegal, está formado por una aplicación web con componentes SIG que ofrecen una mayor y mejor información sobre las parcelas y su entorno. El desarrollo se apoya en el uso de software libre y en la utilización de estándares para el manejo de la información geográfica. El artículo detalla la arquitectura, componentes y funcionalidades del sistema y ofrece datos estadísticos de su utilización.

Palabras clave: Banco de Tierras; SIG-Web; software libre; e-government

\begin{abstract}
This paper describes the information system of the Galician Land Bank. The Galician Land Bank is a public institution that aims to mitigate the land abandonment by promoting the rental of agricultural plots, acting as a mediator between owners and farmers. Within the objectives of the system are to do the internal management of the Bank, to disseminate it among the public and to enable the carrying out of procedures via Internet. The system, available at http://www.bantegal.com/sitegal, is formed by a web application with a GIS framework that offers better information about the plots and their environment. It has been developed using open source software and standards for the management of the geographic information. The paper describes the architecture of the system, its components and its functionalities and also gives some data about its usage.
\end{abstract}

Keywords: Land Bank, Web-GIS; open source software; e-government 


\section{Introducción}

Desde hace años, muchos lugares de Europa están sufriendo procesos de abandono del ámbito rural debido principalmente a la disminución y cambios en la actividad agrícola (Keenleyside \& Tucker, 2010). Este es el caso de Galicia, una comunidad autónoma situada al noroeste de España caracterizada por una estructura de la propiedad con parcelas muy pequeñas y por una tradición de minifundismo. Para frenar este abandono, las explotaciones agrícolas han de adaptarse a la agricultura moderna y tener tamaño suficiente para ser sostenibles. Es pues importante facilitar a los agricultores el acceso a la tierra, pero en algunos casos es complicado debido a que muchos propietarios han emigrado y son difíciles de localizar, y otros muchos tienen apego a sus propiedades, no desean venderlas y desconfían a la hora de arrendarlas. Además, muchas de las parcelas no tienen las condiciones necesarias para que su cultivo sea rentable.

Con el objetivo de mejorar esta situación, el gobierno gallego (Xunta de Galicia), puso en marcha el Banco de Tierras, un instrumento legal para intentar solucionar los problemas mencionados. Para gestionarlo y facilitar los trámites a través de Internet, la Consellería do Medio Rural firmó un convenio con el Laboratorio del Territorio, de la Universidad de Santiago de Compostela, y el Grupo de Arquitectura de Computadores, de la Universidad de A Coruña para la creación de un sistema de información basado en un prototipo desarrollado por ambos grupos en un proyecto previo que pretendía unos objetivos similares (Crecente, Doallo, Miranda, Ónega, Parapar, \& Touriño, 2005). El sistema creado para el Banco de Tierras comparte muchas de las bases de dicho prototipo como son permitir la interacción de los usuarios del Banco, el uso de software libre para abaratar costes y facilitar su implantación y la inclusión de funcionalidades de los SIG para ofrecer una gestión más completa e integrada de las parcelas.

Considerando al Banco de Tierras como principal instrumento de movilidad, estudios recientes (Corbelle-Rico, Crecente-Maseda, \& Santé-Riveira, 2012) indican que desde 1972 hasta 2001 se ha registrado un abandono en Galicia de 70.000 ha; que considerando una superficie media de 0.25 ha/parcela, resultarían unas 280.000 parcelas susceptibles de ser arrendadas.

En este artículo describimos el sistema desarrollado, el cual se encuentra en plena explotación por parte de la administración, y es de acceso público para propietarios, arrendatarios e interesados en general, a través de la dirección http://www.bantegal.com/sitegal. El artículo está estructurado de la siguiente manera: la sección 2 resume el funcionamiento del Banco de Tierras y hace referencia a algunas experiencias similares. La sección 3 proporciona una visión general de las funcionalidades desarrolladas, mientras que la sección 4 se dedica a describir la estructura interna del sistema. En la sección 5 se comentan los resultados obtenidos y se dan datos de uso del Banco de Tierras y del sistema. Finalmente, en la sección 6, se explica en que se está trabajando actualmente y se indican algunas ideas para la evolución del sistema. 


\section{Funcionamiento del Banco de Tierras de Galicia}

El Banco de Tierras de Galicia, en funcionamiento desde noviembre de 2007, es un organismo público dependiente de la Consellería do Medio Rural e do Mar de la Xunta de Galicia. Para su funcionamiento dispone de una oficina central y cuenta con la colaboración de oficinas agrarias comarcales públicas repartidas por todo el territorio gallego, a las que pueden acudir los interesados a realizar los trámites pertinentes. El cometido del Banco es hacer de intermediario en el arrendamiento de parcelas entre propietarios y agricultores. El proceso se divide en dos fases: una fase de incorporación de la parcela al Banco y una fase de arrendamiento.

\subsection{Proceso de incorporación de parcelas al Banco de Tierras}

En principio, cualquier parcela agrícola es susceptible de ser incorporada al Banco. Las parcelas de particulares siguen perteneciendo a sus propietarios pero al incorporarlas, autorizan al Banco a arrendarlas a terceros para usos agrícolas. Mientras no son arrendadas, pueden seguir siendo utilizadas por sus propietarios. El periodo de permanencia en el Banco es indefinido, permanecerán hasta que el propietario solicite su retirada, lo que podrá hacer en cualquier momento salvo que la parcela esté ya arrendada, en cuyo caso deberá esperar a que finalice el contrato de arrendamiento.

Para incorporar una parcela al Banco, el propietario debe realizar una solicitud de incorporación y esta debe ser aceptada, en base a las características de la parcela. El precio de arrendamiento es fijado por el Banco, que establece anualmente unos precios de referencia por unidad de superficie dependiendo del tipo de terreno y de la parroquia en la que esté la parcela. El Banco no se compromete a arrendar las parcelas, dependerá de que existan agricultores interesados. Mientras las parcelas no se arrienden sus propietarios no reciben ningún pago. Si se arriendan, los propietarios recibirán el importe del arrendamiento salvo una comisión del 1\% por gastos de gestión.

Además de las parcelas de particulares, el Banco posee parcelas de titularidad pública, mayoritariamente procedentes de terrenos sobrantes de procesos de concentraciones parcelarias (masas comunes).

\subsection{Proceso de arrendamiento de parcelas del Banco de Tierras}

Cualquier usuario puede consultar la lista de parcelas disponibles en el Banco y pedir su arrendamiento presentando para ello una solicitud oficial. Si sobre una parcela concurre más de una solicitud, la ley establece varios criterios para elegir cual será aceptada. El arrendatario firmará el contrato de arrendamiento con el Banco por una duración de cinco años, aunque si el propietario estuviese de acuerdo el arrendamiento podría durar más. El Banco garantiza al propietario el cobro del arrendamiento de la parcela mientras esta esté arrendada y que cuando finalice, la parcela será devuelta en las mismas condiciones que estaba antes de arrendarse. Una vez finalizado un arrendamiento, la parcela vuelve a estar disponible en el Banco o puede ser retirada por el propietario, si este lo desea. 


\subsection{Experiencias similares}

Actualmente existen diversos lugares donde ya funcionan bancos de tierras, sin embargo, no se han encontrado aplicaciones SIG-Web públicas que permitan la interacción de usuarios con el Banco. Uno de estos casos es el del Banco de Tierras de Asturias, que dispone de una aplicación SIG (Carcedo, Fernández, \& González, 1998) pero sólo para su gestión interna. Otro caso es el de la Diputación Foral de Vizcaya (País Vasco), que ha iniciado los pasos para crear un Fondo de Suelo Agrario. A nivel europeo, el banco de tierras más conocido es la sociedad SAFER, en Francia, que fue creada a principios de los años 6o. Su gestión se basa en la compra-venta de propiedades, teniendo un derecho de tanteo sobre las tierras vendidas. Dispone de sitio web, http://www.proprietes-rurales.com, donde ofertan las propiedades disponibles, pero sin capacidades SIG.

Aparte de los bancos de tierras, existen sitios web desarrollados por iniciativas privadas como http://www.turofincas.com, http://www.buscafincas.com o http://www.fincasysolares.com, que se dedican al negocio de venta y alquiler de parcelas, construcciones y demás propiedades. Fuera de España, existen algunos especializados en las propiedades rurales como http://www.fazonline.com.br, o http://www.landandranchsales.com.

En general, en estos sitios web no se hace uso de entornos SIG para la localización y la consulta de información de las propiedades. Uno de los posibles motivos es el alto coste de obtención de las capas de información y otro es la complejidad de integración de los sistemas SIG en procesos como la compra-venta o el alquiler de tierras. No obstante, esto está cambiando gracias sobre todo a la popularización de servicios de mapas como Google Maps y cada vez son más los sitios que se apoyan en herramientas de terceros para mostrar los lugares en donde tienen sus ofertas. Ejemplos de esto son http://www.housingmaps.com y http://www.farmseller.com.

\section{Visión general y funcionalidades}

El sistema de información ha sido desarrollado para solucionar los problemas y alcanzar los objetivos descritos en las secciones anteriores y se ha convertido en pieza clave para el funcionamiento del Banco de Tierras. Desde su versión inicial, se le han ido añadiendo mejoras y nuevas funcionalidades. Otro de los objetivos que se pretendió desde el inicio fue el uso de software libre. Esto ha permitido un ahorro importante en costes de licencias, tanto para desarrollo como para producción, y también ha facilitado el trabajo al no tener que estar pendientes de las restricciones de instalación que imponen algunas licencias privativas.

El sistema está accesible para cualquier persona, distinguiéndose los siguientes tipos de usuarios:

\section{Usuarios no registrados}

Los usuarios no registrados pueden consultar las parcelas disponibles en el Banco de Tierras, tanto en forma de lista, como sobre un visor web de mapas interactivo (ver Figura 1) donde pueden visualizar la posición y forma exacta de cada parcela sobre una 
ortofotografía de alta resolución. Las características técnicas del visor se detallan en la sección 4.2.

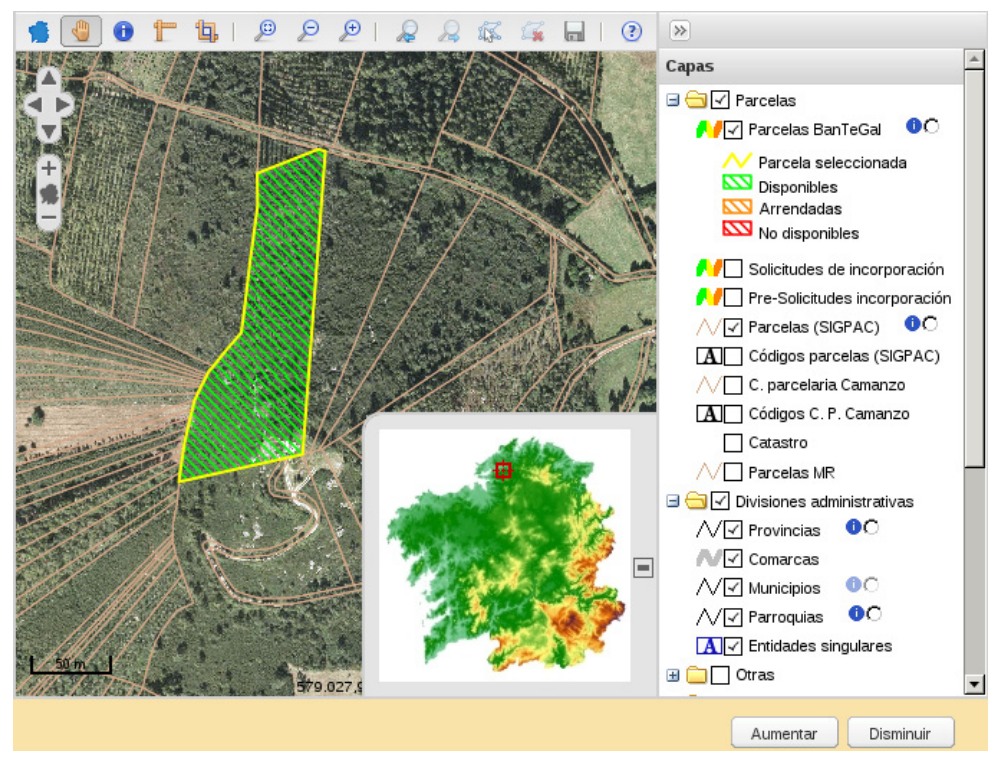

Figura 1 - Visor de mapas con una parcela seleccionada.

Mediante un buscador el usuario también puede buscar las parcelas que le interesen según criterios de superficie, situación, precio, etc. Cuando un usuario no encuentra parcelas que se ajusten a sus necesidades puede introducir en la aplicación las características de las parcelas que necesita y se le suministra un enlace personalizado para que en cualquier momento pueda consultar las que se adapten a sus necesidades. Además, el Banco le enviará un aviso por correo electrónico o SMS cuando se incorpore alguna parcela de su interés.

\section{Usuarios registrados}

Si un usuario desea solicitar el arrendamiento de una parcela del Banco o su incorporación al fondo de tierras, ha de registrarse en el sistema. Para solicitar una incorporación, el usuario puede identificar su parcela de dos modos: o bien moviéndose por el mapa hasta localizarla, o bien introduciendo el código catastral de la parcela y el sistema la buscará automáticamente. Si los límites de la parcela que figuran en el mapa no son correctos, el usuario puede dibujar él mismo la parcela sobre el visor. Para completar la incorporación también ha de introducir los datos de los propietarios. El precio de arrendamiento de la parcela es calculado automáticamente por el sistema en función de la zona en la que se encuentre y los usos a los que esté dedicada. A continuación, el sistema generará automáticamente el documento de solicitud (en formato PDF, DOC u ODT), el cual deberá ser impreso y firmado por los propietarios. Una vez firmado se entregará en alguna oficina junto con la documentación necesaria. Hecho esto, los gestores del Banco la estudiarán y decidirán sobre su aprobación o denegación. 
Si un agricultor encuentra una parcela que le interesa, también puede introducir la solicitud a través de la aplicación, indicando qué condiciones cumple en relación a los distintos criterios que la ley contempla para la selección del arrendatario en caso de que existan varias solicitudes de arrendamiento sobre la misma parcela. El documento de solicitud que se genera también ha de ser firmado y entregado. Los usuarios pueden consultar el estado de sus solicitudes a través de la aplicación.

\section{Gestores del Banco de Tierras}

Los gestores del Banco de Tierras se encargan de estudiar las solicitudes y de aceptarlas o rechazarlas. Existen varios tipos de gestores (comarcales, centrales, administradores) con distintos permisos. Algunas de las funcionalidades a las que tienen acceso son las siguientes: introducción de datos de eventos relacionados con parcelas (visitas realizadas para comprobar su estado, peticiones recibidas para cambios de uso...); generación de estadísticas sobre las parcelas y solicitudes; importación de archivos shape para actualizar geometrías de parcelas; introducción de archivos adjuntos vinculados a solicitudes, parcelas y arrendamientos (contratos escaneados en formato PDF, fotografías, etc.); conexión con servicios web de la oficina del Catastro para la obtención de las geometrías actualizadas de las parcelas y las referencias catastrales.

El sistema también implementa la automatización de diversas tareas como: cálculo de los puntos asignados a las solicitudes de arrendamiento sobre una parcela en base a las características preferentes que reúnen los solicitantes; cálculos de los usos de una parcela mediante operaciones espaciales (intersección de la geometría de la parcela con la capa de usos); cálculo del precio de cada parcela en función de sus usos y situación; actualización de los importes de los arrendamientos de acuerdo con la variación del índice de precios al consumo (IPC) de España.

\section{Implementación del sistema}

Los detalles del sistema desarrollado, junto con sus componentes, fuentes de datos, e información sobre su despliegue son descritos a continuación.

\subsection{Arquitectura global}

En la Figura 2 se muestra un esquema de la arquitectura del sistema. El núcleo es una aplicación web Java que implementa la lógica de las funcionalidades relacionadas con las incorporaciones y arrendamientos de parcelas. Se encarga también de realizar el almacenamiento de la información en base de datos y de generar la interfaz de usuario mediante páginas web dinámicas, las cuales consultan los usuarios a través de sus navegadores. Esta aplicación accede a la información geográfica a través de un servidor de mapas, que es el otro pilar del sistema. El servidor de mapas es un proceso independiente que se encarga de generar los mapas que solicita el usuario y en general actúa de intermediario con la información geográfica, también para peticiones de atributos de los objetos. Así se independiza la aplicación de los orígenes de datos geográficos.

La aplicación Java se encarga de insertar el visor de mapas en aquellas páginas web en las que se usa, particularizándolo según los distintos casos de uso (introducción de 
parcela, consulta de parcela, modificación de geometrías, etc.). Mediante JavaScript, el visor captura las acciones del usuario y las traduce en las correspondientes peticiones al servidor de mapas, con el que se comunica directamente usando técnicas AJAX (Asynchronous JavaScript and XML). Una vez que el servidor responde a una petición, el visor se encarga de actualizar aquellas partes de la página que sea necesario, de manera que al no recargar toda la página, se reduce el tiempo de carga y la interfaz de usuario se hace más amigable. Los mapas se generan en formato raster (JPEG o PNG) para que el tamaño de los archivos a descargar sea el menor posible.

Cuando el usuario selecciona una parcela para solicitar su incorporación al Banco, el visor comunica a la aplicación Java de qué parcela se trata, y la aplicación pide al servidor geográfico los atributos de dicho objeto (superficie, usos, situación...) y se los muestra al usuario en un formulario, pudiendo éste confirmarlos o corregirlos.

Consecuentemente con lo anterior, la información manejada por el sistema también está separada en dos grupos: la información relativa a las parcelas y usuarios del Banco de Tierras, y la información geográfica de referencia, creándose sendas bases de datos (a las que llamaremos base de datos del Banco y base de datos geográfica, respectivamente), ambas con soporte para información espacial. La aplicación Java gestiona la base de datos del Banco mientras que la base de datos geográfica es accedida por el servidor geográfico.

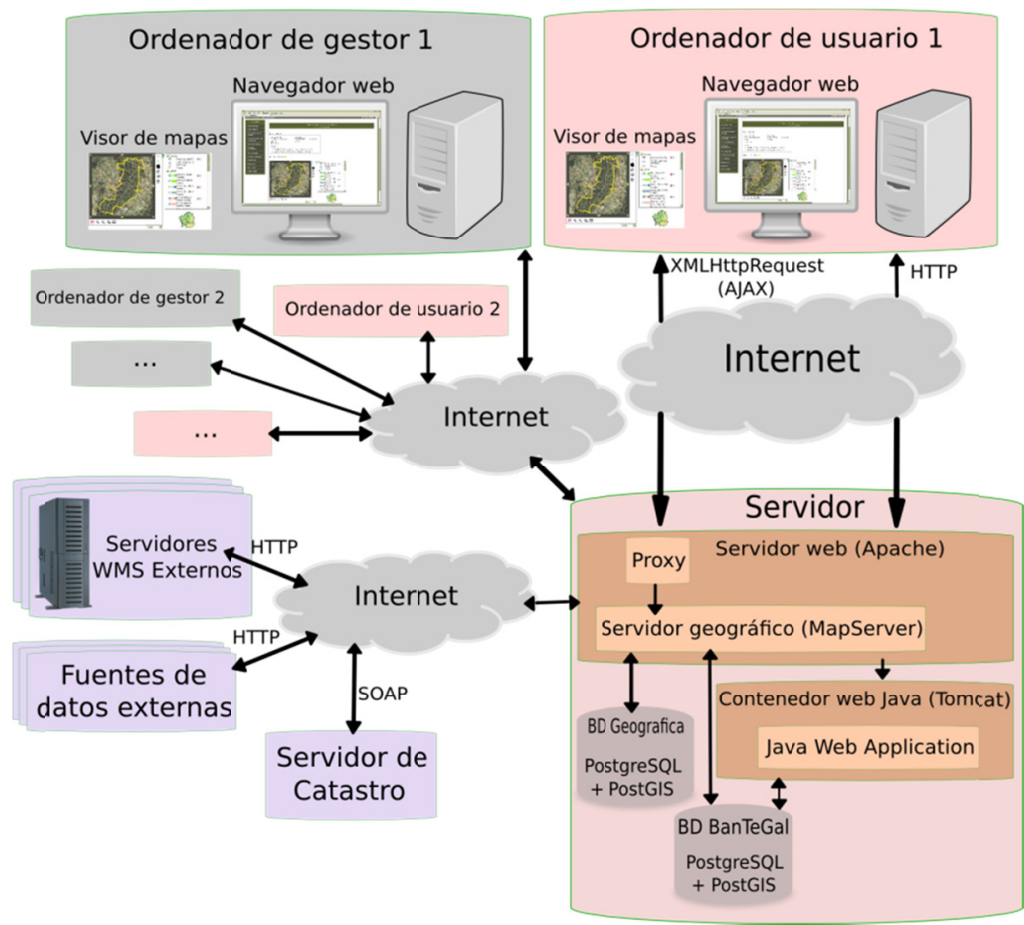

Figura 2 - Arquitectura del sistema. 
Además de la base de datos geográfica, parte de la información geográfica procede de servicios externos a través de los protocolos WMS (Web Map Service) y WFS (Web Feature Service), ambos servicios definidos por el OGC (Open GeoSpatial Consortium), asociación internacional dedicada a la definición de estándares abiertos para la interoperabilidad de sistemas SIG. El WMS se usa para la obtención de mapas con distintas capas de información (normalmente en formato raster) y el WFS sirve para la obtención de datos en formato vectorial junto con los atributos alfanuméricos de los objetos (normalmente en formato GML). Este tipo de servicios encajan dentro de las Infraestructuras de Datos Espaciales (IDE).

\subsection{Componentes del sistema}

A continuación se describe con más detalle cada uno de los componentes del sistema y de las herramientas y librerías utilizadas.

\section{Aplicación web Java}

La aplicación web Java ha sido implementada utilizando herramientas software libre (Tabla 1) y haciendo uso de diversos patrones de diseño (Johnson, Singh, \& Stearns, 2002) mostrados en la Figura 3. Como patrón arquitectónico se ha utilizado estructura en tres capas siguiendo el patrón Modelo-Vista-Controlador (MVC)

Tabla 1 - Herramientas utilizadas en la implementación de la aplicación web.

\begin{tabular}{|c|c|c|}
\hline Nombre & Capa & Descripción \\
\hline Hibernate & Modelo & $\begin{array}{l}\text { Herramienta de transformación objeto-relacional utilizada } \\
\text { para la lectura y escritura en base de datos. }\end{array}$ \\
\hline Spring & Modelo & $\begin{array}{l}\text { Entorno con numerosas funcionalidades para el desarrollo } \\
\text { de aplicaciones web Java. }\end{array}$ \\
\hline Apache Struts & Vista/Controlador & Framework para el desarrollo de aplicaciones web MVC. \\
\hline $\begin{array}{l}\text { JavaServer Pages } \\
\text { (JSP) }\end{array}$ & Vista & $\begin{array}{l}\text { Tecnología Java para el desarrollo de páginas web con } \\
\text { contenido dinámico. }\end{array}$ \\
\hline AjaxTags & Vista & Librería JSP para implementar funcionalidades AJAX. \\
\hline DisplayTag & Vista & $\begin{array}{l}\text { Librería JSP que ayuda a la creación de tablas HTML con } \\
\text { paginación. }\end{array}$ \\
\hline JCaptcha & Vista & Implementación de los conocidos CAPTCHA. \\
\hline JODReports & Controlador & Generador de informes basados en plantillas OpenOffice. \\
\hline Quartz Scheduler & Modelo & $\begin{array}{l}\text { Conjunto de librerías Java que permiten la ejecución de } \\
\text { tareas de manera asíncrona, automática y programada. }\end{array}$ \\
\hline
\end{tabular}




\begin{tabular}{lll}
\hline ClamAV & Modelo & $\begin{array}{l}\text { Antivirus que puede ser utilizado en aplicaciones web a } \\
\text { través de su API. }\end{array}$ \\
\hline Java Topology Suite & Modelo & $\begin{array}{l}\text { Librería que implementa multitud de operaciones } \\
\text { espaciales bidimensionales (insercciones, uniones...). }\end{array}$ \\
\hline GeoTools & Modelo & $\begin{array}{l}\text { Librería que ofrece diversos mecanismos para el manejo } \\
\text { de datos espaciales. Es utilizada para la lectura de archivos } \\
\text { de datos con formato SHP. }\end{array}$ \\
\hline
\end{tabular}

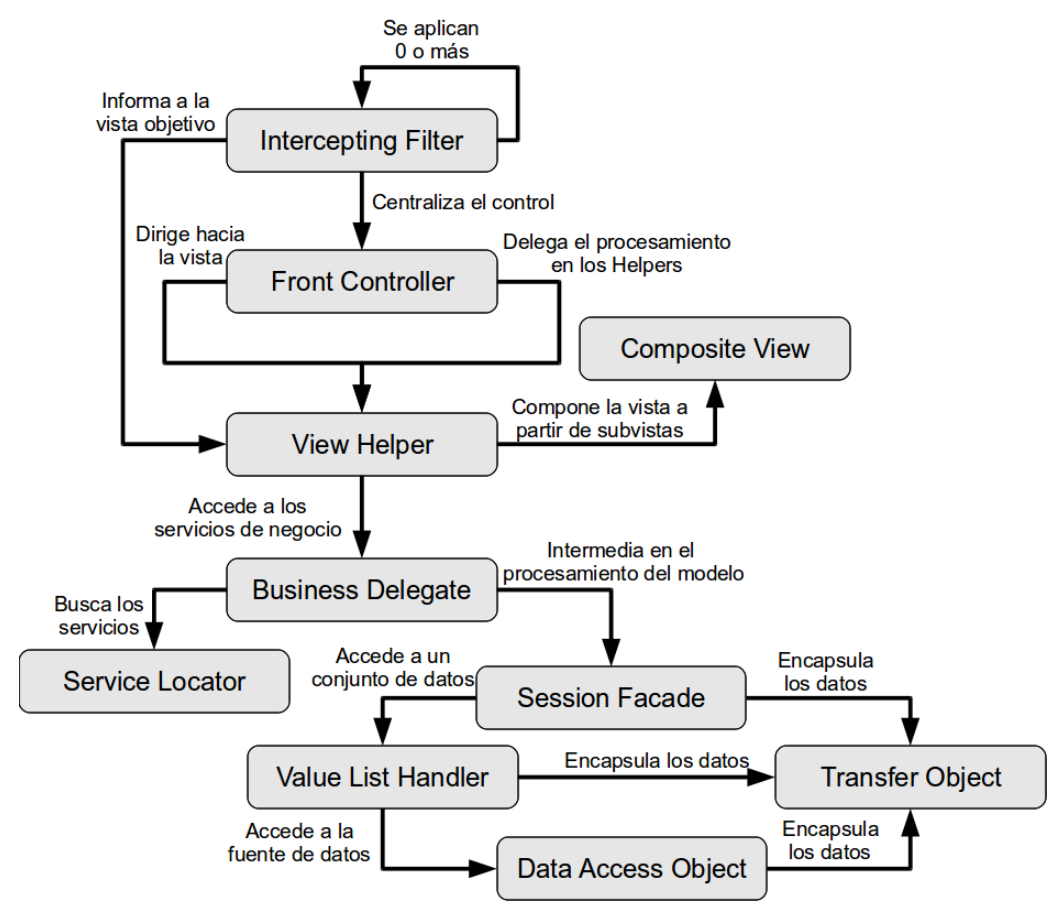

Figura 3 - Patrones de diseño utilizados.

\section{Visor de mapas}

El visor de mapas (ver Figura 1) está basado en OpenLayers. OpenLayers es el visor libre más popular en la actualidad. Se trata de una aplicación JavaScript que hace uso intensivo de AJAX y entre sus principales características están el que permite el acceso a muchos tipos de servicios de datos, tanto estándar (WMS, WFS, WMTS, KML, GML...) como no estándar (Google Maps, Yahoo Maps, OpenStreetMap...). Por contra, su interfaz de usuario es algo pobre, aunque gracias a las posibilidades de ampliación y personalización que ofrece existen complementos centrados en su mejora. En nuestro caso usamos GeoExt, basado en la librería Ext. Entre las funcionalidades del visor 
están: desplazamiento por el mapa y zooms; medición de longitudes y áreas; consulta de información sobre los objetos visibles; selección y centrado de objetos; link para recuperar la situación actual del mapa en cualquier momento; digitalización y modificación de geometrías; etc.

\section{Servidor de mapas}

Como servidor de mapas se usa MapServer. Se trata de una de las aplicaciones SIG libres más veteranas y populares. Está programado en lenguaje $\mathrm{C}$ y funciona como un CGI acoplado al servidor web, aunque también ofrece APIS para numerosos lenguajes. Tiene un buen rendimiento y destaca por soportar numerosas fuentes de datos y estándares. En particular, permite el acceso en cascada a otros servidores WMS y WFS.

\section{Almacenamiento}

Como servidor de datos se usa PostgreSQL con su extensión espacial PostGIS. Su elección se debe a que PostGIS es el software libre que mejor soporta el estándar del OGC para bases de datos espaciales. De hecho está certificado por dicho organismo como acorde al perfil Types and Functions de la especificación Simple Features Specification for SQL. Posee una buena capacidad de indexación espacial y está soportado por la mayoría de servidores de mapas.

\section{Integración de componentes}

La conexión de la aplicación Java con la base de datos se hace mediante Hibernate e Hibernate Spatial los cuales soportan PostgreSQL y PostGIS. Una posible migración a cualquiera de los servidores de base de datos soportados por estas herramientas sería factible. De la conexión de MapServer con la base de datos se ocupa el propio MapServer, siendo también posible migrar a otra base de datos (MapServer soporta varios servidores de bases de datos espaciales) e incluso a otro servidor de mapas (PostgreSQL+PostGIS es soportado por la mayoría de servidores de mapas).

La comunicación entre el visor y la aplicación Java con el servidor de mapas es la parte que ha requerido mayor trabajo. Si bien la visualización de capas en el visor es una tarea sencilla, existen casos de uso que requieren una comunicación en varios pasos entre ambas partes. Por ejemplo, para introducir una solicitud de incorporación de una parcela los pasos son los siguientes: el usuario sitúa el mapa en la zona donde se encuentra la parcela (peticiones WMS GetMap para dibujar el mapa); selecciona una parcela y esta se resalta dibujando los bordes en otro color (petición WMS GetFeatureInfo para identificar qué parcela es y petición WMS GetMap para obtener el mapa con la parcela resaltada); y confirma que la parcela resaltada es la que quiere incorporar y esta se guarda en la base de datos (petición WFS GetFeature para obtener la geometría en formato vectorial y los atributos alfanuméricos de la parcela).

El diseño utilizado permite que todos los componentes del sistema sean independientes entre sí. Así, la sustitución de cualquiera de ellos sería transparente para el resto del sistema. 


\subsection{Fuentes de datos geográficos}

Una de las cosas más importantes para que el sistema cumpla con los objetivos propuestos y tenga una buena acogida es que los datos geográficos utilizados posean suficiente calidad. En particular, hay tres capas de información que son básicas para el funcionamiento del sistema: ortofografías del terreno, usos del suelo y la división parcelaria. En España, afortunadamente, se dispone de buenas fuentes de información para las tres.

Con respecto a las ortofotos, en el marco del Plan Nacional de Ortofografía Aérea, cada 2 años se vuela sobre toda España y se generan ortofotos con una resolución que alterna entre de 25 y $50 \mathrm{~cm} /$ píxel. Con respecto a las parcelas y a los usos del suelo, el SIGPAC (Sistema de Información de Parcelas Agrícolas), sistema encargado de la gestión de las ayudas agrícolas de la Unión Europea, dispone de ambas capas de información en formato vectorial para todo el país y las actualiza periódicamente. Para usar las capas de parcelario y los usos del suelo se han copiado los datos en la base de datos local debido a que no existen servidores WFS disponibles que permitan acceder a sus atributos. De las ortofotos no se tiene copia local, pues se usan únicamente como fondo del mapa y sí existen varios servidores WMS de acceso público que las suministran.

Otros datos usados son las divisiones administrativas, que proceden del Sistema de Información Territorial de Galicia (SITGA), y las zonas naturales protegidas que se obtiene del servidor WMS del Ministerio de Medio Ambiente.

Dada la estructura modular del sistema y gracias al uso de estándares, la incorporación al visor de mapas de nuevas capas de información disponibles vía WMS resulta muy sencilla.

\subsection{Despliegue del sistema}

El sistema es multiplataforma (Linux, Windows, Mac OS X) ya que todos sus componentes lo son (PostgreSQL, Java, MapServer). Actualmente se encuentra instalado en dos máquinas virtuales con sistema operativo SUSE Linux Enterprise Server 10 SP1, 4GB de RAM, y un procesador Intel Xeon X5450 a 3.00GHz con dos núcleos. En una de las máquinas está el servidor web (Apache), el servidor de mapas y la base de datos geográfica. En la otra se encuentra la aplicación web en un servidor Tomcat y la base de datos del Banco. Los usuarios acceden al servidor Apache de la primera máquina la cual redirige las peticiones correspondientes al servidor Tomcat de la segunda máquina, la cual sólo es accesible desde la red interna.

La tabla 2 resume las principales tecnologías utilizadas en el sistema. 
Tabla 2 - Resumen de tecnologías utilizadas.

\begin{tabular}{|c|c|c|}
\hline Elemento & Recurso & Breve descripción \\
\hline \multirow[t]{2}{*}{ Servidor web } & Apache HTTP & Servidor HTTP. \\
\hline & Server & \\
\hline Contenedor web & Apache Tomcat & Contenedor de aplicaciones web Java. \\
\hline Servidor de mapas & MapServer & Servidor geográfico con soporte para servicios OGC. \\
\hline Base de datos & PostgreSQL & Sistema gestor de bases de datos relacionales. \\
\hline Extensión geográfica & PostGIS & Extensión con capacidades espaciales para PostgreSQL. \\
\hline \multirow[t]{5}{*}{ Estándares OGC } & WMS & Web Map Service: servicio de imágenes georreferenciadas. \\
\hline & WFS & Web Feature Service: servicio de datos geográficos. \\
\hline & GML & Geography Markup Language: gramática XML para \\
\hline & & expresar datos y objetos geográficos. \\
\hline & SLD & $\begin{array}{l}\text { Styled Layer Descriptor: describe la apariencia de las } \\
\text { capas de información. }\end{array}$ \\
\hline \multirow{2}{*}{$\begin{array}{l}\text { Formato de datos } \\
\text { geográficos }\end{array}$} & Shapefile & Formato de datos vectoriales. \\
\hline & WKT & $\begin{array}{l}\text { Well-Known Text: formato para representar la geometría } \\
\text { de objetos en formato vectorial. }\end{array}$ \\
\hline \multirow{7}{*}{$\begin{array}{l}\text { Fuentes de datos } \\
\text { externos }\end{array}$} & SOAP DGC & Servicio web de la Dirección General de Catastro. \\
\hline & WFS DGC & Servidor WFS de la Dirección General de Catastro. \\
\hline & SIXPAC WMS & Servidor WMS del Sistema de Información de Parcelas \\
\hline & & Agrícolas de Galicia. \\
\hline & SITGA WMS & Servidor WMS del Sistema de Información Territorial de \\
\hline & & Galicia. \\
\hline & PNOA WMS & Servidor WMS del Plan Nacional de Ortografías Aéreas. \\
\hline
\end{tabular}

\section{Resultados y discusión}

El Banco de Tierras está operativo desde finales del año 2007. A fecha de diciembre de 2011, el número de parcelas disponibles en el Banco era de 7.160, las cuales suman un total de 3.049,36 hectáreas. De ellas, se encuentran arrendadas 1.404 parcelas sumando 1.359,80 hectáreas, es decir, un 44,59\% de la superficie de parcelas del Banco está arrendada. Con respecto a los clientes del Banco, el 33,93\% de las solicitudes de 
arrendamiento y el 27,09\% de las solicitudes de incorporación las inician a través del sistema vía Internet. Aunque sería deseable un porcentaje mayor, es un dato muy aceptable debido al perfil de los usuarios: gente de ámbito rural, de edad mediana o avanzada, con formación y experiencia informática reducida y con acceso a Internet de mala calidad, lo que ha sido identificado como una de las grandes dificultades en estas iniciativas rurales (Bayfield, y otros, 2005).

Las consultas espaciales suelen ser las operaciones más costosas en este tipo de sistemas. La tabla 3 muestra tiempos de ejecución medios de las peticiones más frecuentes sobre el servidor de mapas. Se ha probado con distinto número de peticiones por segundo y para cada caso se ha repetido la prueba varias veces (tandas) y se ha hecho la media. Las peticiones probadas han sido para zonas de 1000x1000 metros: consulta WMS GetMap sobre la capa de usos del suelo para un tamaño de mapa de 1024X1024 píxeles; consulta WMS GetFeatureInfo sobre la capa de parcelas para un tamaño de mapa de 1024X1024 píxeles; y consulta WFS GetFeature sobre la capa de usos del suelo utilizando un filtro de tipo bounding box (BBOX).

Tabla 3 - Tiempos medios de ejecución de consultas (en segundos).

\begin{tabular}{lccccc}
\hline Petición & Una petición & 5 por seg. & 10 por seg. & 2o por seg. & 50 por seg. \\
\hline GetMap & 1,10 & 2,21 & 3,76 & 6,38 & 13,81 \\
\hline GetFeatureInfo & 0,64 & 1,46 & 2,53 & 4,31 & 10,14 \\
\hline GetFeature & 1,18 & 2,12 & 3,31 & 5,85 & 11,81 \\
\hline
\end{tabular}

Las parcelas y usos del suelo están almacenados en local y suman aproximadamente 12.000.000 y 16.000.000 registros respectivamente. Como se puede ver, para un número de usuarios moderado, los tiempos de ejecución son bastante buenos, dado el gran volumen de datos que involucran. En caso de necesitar más rendimiento, el sistema está diseñado para poder replicarse y utilizar balanceadores de carga sin necesidad de tocar el código fuente, sólo los archivos de configuración.

\subsection{Experiencia con los usuarios}

La comunicación permanente y directa entre los desarrolladores del sistema y los usuarios del Banco ha hecho que la aplicación esté en un ciclo de mejora continua y de ampliación de sus funcionalidades. Además, se han adaptado tecnologías y procedimientos para facilitar la usabilidad del sistema. Por ejemplo, en su momento se detectó que existían demandantes de parcelas que no encontraban en el Banco parcelas que se ajustasen a sus necesidades. Esto llevó a desarrollar una funcionalidad que permite al usuario introducir las características de las parcelas deseadas, y cuando se incorpore alguna parcela al Banco que cumpla con dichas características se avisa al demandante a través de SMS o correo electrónico.

Parte de los usuarios potenciales del sistema son gente escasos conocimientos en nuevas tecnologías e incluso un acceso limitado a Internet. Es por esto que los procesos de introducción y arrendamiento se han dividido en pasos guiados y detallados para 
que resulte fácil e intuitivo realizarlos. Tampoco es necesaria la instalación de ningún plugin en el navegador del usuario (como puede suceder con otros visores geográficos) y se ha tratado de optimizar el sistema para que consuma poco ancho de banda.

La comunicación a través del sistema de información entre los gestores del Banco, sobre todo entre distintos roles (por ejemplo entre gestores centrales y gestores de las oficinas comarcales) es una necesidad que surgió cuando el Banco ya estaba en pleno funcionamiento. Por eso se ha creado un foro interno para gestores y un sistema de alertas y noticias. Con vistas a dinamizar el uso de la web, y en general del Banco, se está planteando la extensión de estas funcionalidades a todos los clientes y visitantes anónimos.

\section{Conclusiones y trabajo futuro}

El concepto de Banco de Tierras es novedoso. El desarrollo de su sistema de gestión, aquí explicado, es pues innovador y ha supuesto un reto ya que tanto el Banco como su aplicación SIG-Web han ido evolucionando conjuntamente. Los resultados obtenidos en estos ya más de cuatro años en funcionamiento, son muy satisfactorios. El sistema cumple con los objetivos para los que ha sido desarrollado y es la pieza clave para la gestión del Banco de Tierras. La gran cantidad de información manejada por el sistema está bien gestionada gracias a la utilización de estándares. El uso de software libre se ha mostrado suficientemente robusto, con buen rendimiento y ha ayudado a abaratar los costes. Además, ha tenido una acogida aceptable entre usuarios en principio poco habituados al uso de aplicaciones informáticas.

Algunas ideas para mejorar la aplicación son: opción para añadir al visor otras capas WMS y poder visualizarlas simultáneamente con el resto de capas; uso de tiles (división del mapa en celdas) para que las peticiones se hagan tile a tile y así la carga del mapa sea más dinámica; actualización del framework web utilizado (Struts) por uno más moderno (Wicket), cuya integración ya sería en sí un desafío interesante; implementación de opciones para introducción y arrendamiento agrupado de parcelas; integración con la gestión de concentraciones parcelarias; intermediación para el arrendamiento de explotaciones agrarias.

\section{Agradecimientos}

En este trabajo han colaborado y ha sido financiado por la Consellería do Medio Rural e do Mar de la Xunta de Galicia, la Sociedad Anónima Gestora Bantegal y la Agencia Gallega de Desarrollo Rural. También está incluido en el proyecto Sistema de Información Geográfico para la Planificación Urbanística y Ordenación del Territorio utilizando Técnicas de Optimización en Procesadores Multi-núcleo (ref. o8SINo11291PR).

\section{Referencias bibliográficas}

Bayfield, N. G., Conroy, J., Birnie, R. V., Geddes, A., Midgley, J. L., Shucksmith, M. D., et al. (2005). Current awareness, use and perceived priorities for rural databases in Scotland. Land Use Policy, 22(2), 153-162. 
Carcedo, L., Fernández, M. B., \& González, V. (1998). Intergraph - Aplicación de tecnologías abiertas a la gestión territorial de la comisión regional del Banco de Tierras Principado de Asturias. Mapping Interactivo(49).

Corbelle-Rico, E., Crecente-Maseda, R., \& Santé-Riveira, I. (2012). Multi-scale assessment and spatial modelling of agricultural land abandonment in a European peripheral region: Galicia (Spain). Land Use Policy, 29(3), 493-501.

Crecente, R., Doallo, R., Miranda, D., Ónega, F., Parapar, J., \& Touriño, J. (2005). Tecnología SIG y web para la dinamización del mercado de tierras en Galicia. III Jornadas Sindur. Sociedad de la Información en Espacios Periféricos, Nuevas Formas de Exclusión Social (pp. 199-210). Servicio de Edición Digital de la Universidad de Santiago de Compostela.

Johnson, M., Singh, I., \& Stearns, B. (2002). Designing Enterprise Applications with the J2EE Platform (2nd ed.). Prentice Hall PTR.

Keenleyside, C., \& Tucker, G. (2010). Farmland Abandonment in the EU: an Assessment of Trends and Prospects. Institue for European Environmental Policy, London. 